\title{
ON HIGHER-DIMENSIONAL FIBERED KNOTS
}

\author{
BY

\section{J. J. ANDREWS AND D. W. SUMNERS( $\left.{ }^{1}\right)$}

\begin{abstract}
The geometrical properties of a fibration of a knot complement over $S^{1}$ are used to develop presentations for the homotopy groups as modules over the fundamental group. Some homotopy groups of spun and twist-spun knots are calculated.
\end{abstract}

I. Introduction. Let $K^{n}$ be a smooth submanifold of $S^{n+2}$ which is homeomorphic to $S^{n}$. The pair $\left(S^{n+2}, K\right)$ is a knot, with complement $S=S^{n+2}-K$. A tubular neighborhood of $K$ in $S^{n+2}$ provides us with a map $p: S \rightarrow S^{1}$, which in some cases is a fiber map [7]. When the complement fibers, the homotopy exact sequence of the fibration $F^{n+1} \rightarrow S \stackrel{p}{\rightarrow} S^{1}$ tells us that $\Pi_{i}(F) \cong \Pi_{i}(S), i \geqq 2$, $\Pi_{1}(F) \cong\left[\Pi_{1}(S), \Pi_{1}(S)\right]$ the commutator subgroup of $\Pi_{1}(S)$, and $\Pi_{1}(S) \cong \Pi_{1}(F) \times Z$ the semidirect product of $\Pi_{1}(F)$ and the integers.

If $\Pi_{1}(S)=Z$, and $n \geqq 4$, a necessary and sufficient condition that a fibration exists is that the groups $\Pi_{i}(S)$ are finitely generated as abelian groups for all $i$ [3]. In this case the fiber is simply connected. If $\Pi_{1}(S) \neq Z$, there are certain obstructions which determine whether or not $S$ fibers over the circle [5].

There are many interesting cases where $S$ fibers when $\Pi_{1}(S) \neq Z$, and the fact that $\Pi_{1}(F) \neq 1$ can be used to obtain information about the groups $\Pi_{i}(S)$ and about the homotopy type of $\tilde{S}$, the universal cover of $S$. §II of this paper studies the general situation of a fiber bundle $F^{n+1} \rightarrow S \rightarrow S^{1}$ where $\Pi_{1}(F) \neq 1$ and $F \simeq \bar{F}$, a smooth compact bounded manifold with $\partial \bar{F}$ homeomorphic to $S^{n}$.

In §III we study the particular case of fibered $k$-spun knots. If a knot fibers, then the $k$-spin of the knot fibers by $k$-spinning the fibration. For a knot of $S^{1}$ in $S^{3}$, the $k$-spun knot fibers if and only if the knot of $S^{1}$ in $S^{3}$ fibers. In this case, no matter which two (nontrivial) Neuwirth knots one $k$-spins, the universal covers of the resulting knot complements are found to be homotopy equivalent.

In §IV we develop an algebraic result about extending module presentations. If

$$
1 \longrightarrow H \stackrel{\alpha}{\longrightarrow} G \underset{\gamma}{\stackrel{\beta}{\rightleftarrows}} T \longrightarrow 1
$$

Received by the editors September 15, 1969.

AMS 1970 subject classifications. Primary 57C45, 55A25.

Key words and phrases. Fibered knots, twist-spun knots, module structure of homotopy groups.

( ${ }^{1}$ ) This work was partially supported by NSF grant GP-8417.

Copyright (C) 1971, American Mathematical Society 
is an exact sequence of groups such that $\beta \gamma=$ identity on $T$ ( $G$ is then the semidirect product of $H$ and $T$ ), and $A$ is a finitely presented $Z H$-module $(Z H=$ integral group ring of $H$ ) such that $T$ acts on $A$, then the given presentation for $A$ as a $Z H$-module can be extended to a presentation of $A$ as a $Z G$-module. This theorem is then used to determine the structure of the groups $\left\{\Pi_{i}(S)\right\}$ as $Z \Pi_{1}(S)$ modules and the groups $\left\{H_{i}\left(S^{*} ; Z\right)\right\}$ as $\Lambda$-modules, where $S^{*}=$ infinite cyclic cover of $S$ and $\Lambda=$ integral group ring of $J(t)$, the infinite cyclic group of covering transformations of $S^{*}$ generated by $t$.

In $\S \mathrm{V}$ we apply the results of $\S \mathrm{IV}$ to calculate the homotopy structure of simple fibered knots. A fibered $\operatorname{knot}\left(S^{n+2}, K^{n}\right)$ is said to be simple if $\Pi_{i}(S)=0,2 \leqq i \leqq n-1$. Any fibered $\left(S^{4}, K^{2}\right)$ knot is said to be simple. For example, any $k$-spun Neuwirth knot is simple by a theorem of Epstein [4]. For simple knots, complete results are obtained for $\Pi_{n}(S)$ as a $Z \Pi_{1}(S)$-module when the commutator subgroup $\left[\Pi_{1}(S), \Pi_{1}(S)\right]$ is a finite group.

In $§ V I$ we calculate as examples the homotopy structure of the 5-twist-spun trefoil and the $k$-spun trefoil.

We should like to thank the referee for helpful comments, in particular for suggestions simplifying the proofs of Theorems 1 and 9.

II. General theorems on fibered knots. Consider the bundle $F^{n+1} \rightarrow S \rightarrow S^{1}$ where $S=S^{n+2}-K^{n}$ a knot complement, $n \geqq 2$. Let $\approx$ denote diffeomorphism, $\simeq$ denote homotopy equivalence, and $\bigvee$ denote wedge product of spaces. Let $\tilde{S}, \tilde{F}$ denote the universal covers of $S$ and $F$. Clearly $\tilde{S} \approx \widetilde{F} \times R^{1} \simeq \tilde{F}$. Let $G=\Pi_{1}(F)$. and $1_{G}$ denote the identity of $G$. Let $V^{+}=\bigvee_{g \in G} K_{g}^{n}$ be the wedge product of copies of $K$ indexed by $g \in G$ with the identification topology.

Now the fiber $F$ deformation retracts to $\bar{F}$, a compact bounded $(n+1)$-manifold with $\partial \bar{F} \approx K$. The universal cover $\tilde{F}$ induces $\bar{F}^{\sim}$ over $\bar{F}$, and $\tilde{F}$ deformation retracts to $\bar{F}^{\sim}$. We have $\partial \bar{F}^{\sim}=\bigcup_{g \in G} K_{g}^{n}$ the disjoint union of copies of $K$ indexed by $G$, the group of covering translations of $\bar{F}^{\sim}$.

THEOREM 1. If $G=\Pi_{1}(F)$ is not a finite group, then

(i) $\Pi_{i}(F) \cong \Pi_{i}\left(\bar{F}^{\sim}\right) \cong \Pi_{i}\left(\partial \bar{F}^{\sim}\right) \oplus \Pi_{i}\left(\bar{F}^{\sim}, \partial \bar{F}^{\sim}\right)$ as $Z G$-modules, $i \geqq 2$,

(ii) $\Pi_{i}\left(V^{+}\right)$is a direct summand of $\Pi_{i}(S)$ as an abelian group for all $i$.

Proof. There is a retraction $r: \bar{F}^{\sim} \rightarrow \partial \bar{F}^{\sim}$ because the obstructions lie in $H^{i+1}\left(\bar{F}^{\sim}, \partial \bar{F}^{\sim} ; \Pi_{i}\left(\partial \bar{F}^{\sim}\right)\right)$ which vanish for $i<n$ because $\Pi_{i}\left(\partial \bar{F}^{\sim}\right)=0$ in this range, and for $i=n$ because $\bar{F}^{\sim}$ is not compact if $G$ is not finite. The retraction commutes with covering translations in $\bar{F}^{\sim}$, so (i) is proved.

Choosing a base point $* \in K_{1_{g}}$, let $*_{g} \in K_{g}$ represent points in the same fiber as $*$, and choose arcs $a_{g} \subset \bar{F}^{\sim}$ from $*$ to $*_{g}$, such that $a_{g_{1}} \cap a_{g_{2}}=*$ if $g_{1} \neq g_{2}$, and such that the projection of $a_{g}$ in $F$ is in the homotopy class of $g \in G$. Let $\tilde{V}+=\partial \bar{F}^{\sim} \cup$ $\left(\bigcup_{g \in G} a_{g}\right)$. Then $\tilde{V}^{+} \simeq V^{+}$by shrinking the arcs.

There is a retraction from $\bar{F}^{\sim}$ to $\tilde{V}^{+}$for the same reasons as before, only this time the splitting works at the abelian group level. 
Now, let $V=\bigvee_{g \in\left\{G-1_{G}\right\}} K_{g}^{n}$ be the wedge product of copies of $K$ indexed by elements in the set $\left\{G-1_{G}\right\}$. ( $V^{+} \simeq V$ iff $G$ is infinite.)

THEOREM 2. If $G$ is a finite group then $\tilde{S} \simeq Q \vee V$ where $Q$ is a simply-connected finite similicial complex of dimension less than or equal to $n$.

Proof. $\bar{F}^{\sim}$ is compact since $G$ is finite, and $\bar{F}^{\sim}=\bigcup_{i=1}^{|G|} K_{i}$ the finite union of copies of $K$, where $|G|=$ order of $G$ and $K_{1}=K_{1_{a}}$. Choose arcs $a_{i}$ from $* \in K_{1}$ to $*_{i} \in K_{i}$ as in the proof of Theorem 1. We will deformation retract away from $K_{1}$ and isolate $K_{2}$ in a wedge product decomposition for $\bar{F}^{\sim}$. That is, we will obtain a homotopy equivalence $\bar{F}^{\sim} \simeq K_{2} \vee Q_{2}$ where $Q_{2}$ is a bounded $(n+1)$-manifold with $\partial Q_{2} \approx K_{1} \# K_{2}$ (connected sum).

Collapse away along a small tubular neighborhood of $a_{2}$ until the boundary of a small collar neighborhood of $K_{2}$ is reached [Figure 1].

$K_{2}$ is a homotopy sphere, so the collar now deformation retracts from an $n$-disc $D_{2}^{n}$ on its boundary component to $K_{2} \cup \operatorname{arc} \cup L_{2}$ where $L_{2} \approx K_{2}-\operatorname{Int} D_{2}^{n}$, and the
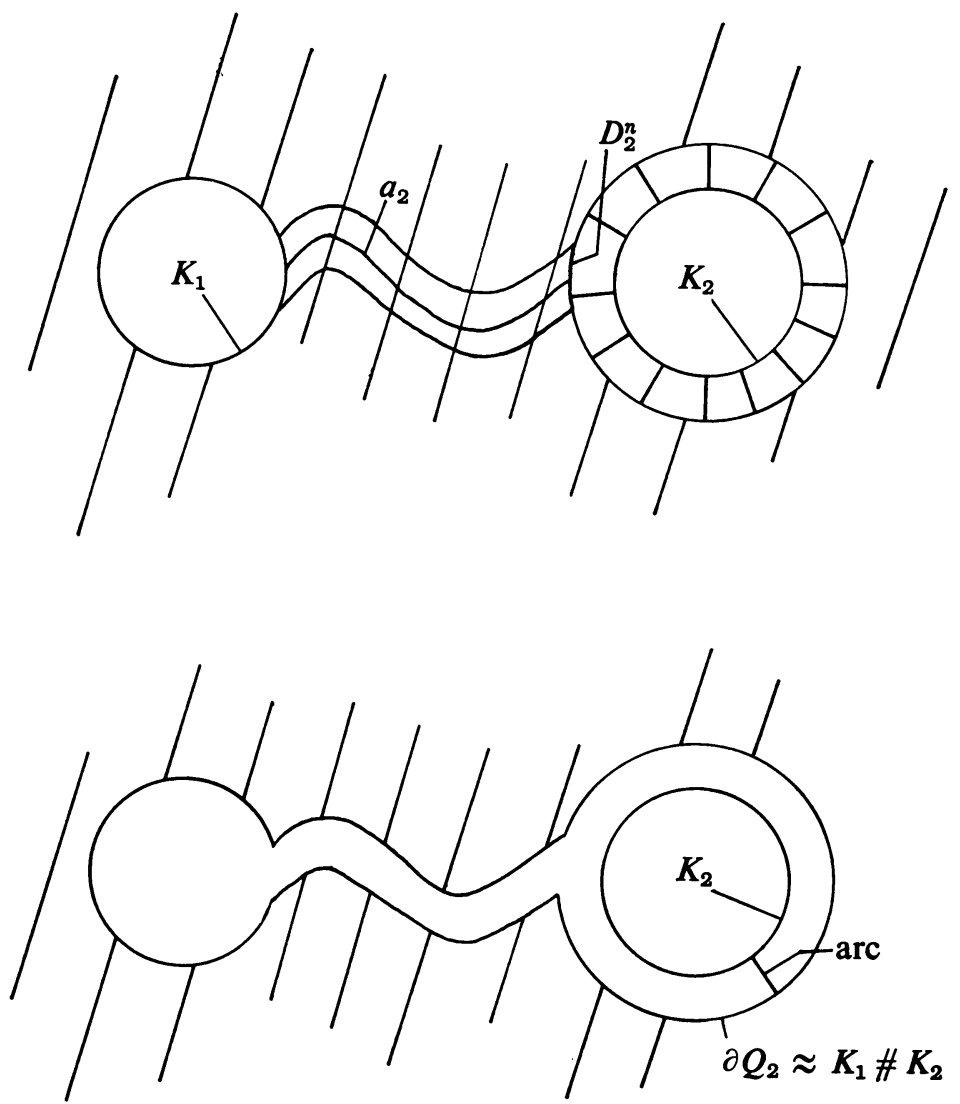

FIGURE 1 
arc is "straight" in the product structure of the collar. Continuing this process, we obtain $\left.K \simeq Q^{\prime} \vee \bigvee\right|_{i=2} ^{|G|} K_{i}=Q^{\prime} \vee V$ where $Q^{\prime}$ is a bounded $(n+1)$-manifold with $\partial Q^{\prime} \approx \#_{i=1}^{G \mid} K_{i}$. Clearly then $K \simeq Q \vee V, Q$ a connected simply-connected $n$-complex obtained from $Q^{\prime}$ by collapsing out all the top-dimensional cells.

COROLlaRY 3. $G$ a finite group and $S$ a simple fibered knot then $\tilde{S} \simeq V$.

Proof. From the proof of Theorem 2 we have $\bar{F}^{\sim} \simeq Q^{\prime} \vee V . Q^{\prime}$ is contractible since $S$ is a simple knot.

We would also note that if $G$ is a finite group, then from Serre's theorem [6, p. 509] we have that $\Pi_{i}\left(\bar{F}^{\sim}\right) \cong \Pi_{i}(S)$ is a finitely generated abelian group for all $i \geqq 2$, since $\bar{F}^{\sim}$ compact.

III. Fibered spun knots. Let $f: B^{n} \rightarrow B^{n+2}$ be a smooth proper embedding $(n \geqq 1)$ of balls. We obtain the $\operatorname{knot}\left(S^{n+2+k}, K^{n+k}\right)$ by $k$-spinning $\left(B^{n+2}, f\left(B^{n}\right)\right)$ in the following manner [4]:

$$
\begin{gathered}
S^{n+2+k}=\left(S^{k} \times B^{n+2}\right) \bigcup_{S^{k} \times f\left(S^{n-1}\right)}\left(B^{k+1} \times S^{n+1}\right), \\
S^{n+k} \approx K^{n+k}=\left(S^{k} \times f\left(B^{n}\right)\right) \bigcup_{S^{k} \times f\left(S^{n-1}\right)}\left(B^{k+1} \times f\left(S^{n-1}\right)\right) .
\end{gathered}
$$

Note. We allow the boundary sphere pair of the ball pair to be knotted.

LEMMA 4. $\left(S^{k+3}, K^{k+1}\right)$ a k-spun $\left(B^{3}, f\left(B^{1}\right)\right)$ fibers iff $\left[\Pi_{1}(B), \Pi_{1}(B)\right]$ is finitely generated, where $B=B^{3}-f\left(B^{1}\right)$.

Proof. In this case $\Pi_{1}(S)=\Pi_{1}(B)$ where $S=S^{k+3}-K^{k+1}$. If $S$ fibers over $S^{1}$, then clearly $\left[\Pi_{1}(B), \Pi_{1}(B)\right]$ is finitely generated. Now $\left(B^{3}, f\left(B^{1}\right)\right)$ determines a unique sphere pair $\left(S^{3}, f^{\prime}\left(S^{1}\right)\right)$, and by the Neuwirth-Stallings Theorem [7, p. 475], $S^{3}-f^{\prime}\left(S^{1}\right)$ fibers if $\left[\Pi_{1}(S), \Pi_{1}(S)\right] \cong\left[\Pi_{1}(B), \Pi_{1}(B)\right]$ is finitely generated. One obtains the fibration for $S$ by $k$-spinning the fibration of the ball pair. Twist spinning a fibered knot to obtain a fibration is described in detail by Zeeman [7]. In order to obtain a fibration by spinning only, one neglects to twist as the spinning goes on.

This process is considered in greater detail in the proof of Theorem 6. Lemma 4 says that if $\left(S^{k+3}, K^{k+1}\right)$ is a $k$-spun fibered knot, then in fact it is a $k$-spun Neuwirth knot. As before, let $V^{+}=\bigvee_{g \in G} S^{k+1}$.

TheOREM 5. Let $\left(S^{k+3}, K^{k+1}\right)$ be a $k$-spun Neuwirth knot of genus $g \geqq 1$. Then $\tilde{S} \simeq V^{+} \vee V^{+} \vee \cdots \vee V^{+}(2 g$-fold wedge products $)$.

Proof. We will study carefully the geometry of the spun fibration. Figure 2 describes the fibration of $B^{3}-f\left(B^{1}\right)$ by $M_{g}^{2}$ the "partially-bounded" punctured torus of genus $g$ [Figure 2].

$M_{g, \phi}^{2}$ is the fiber at time $\phi \in S^{1} . \partial M_{g, \phi}^{2}$ is an open arc lying on $\partial B^{3}$, and is the fiber of the bundle induced on $S^{2}-f\left(S^{0}\right)$. 


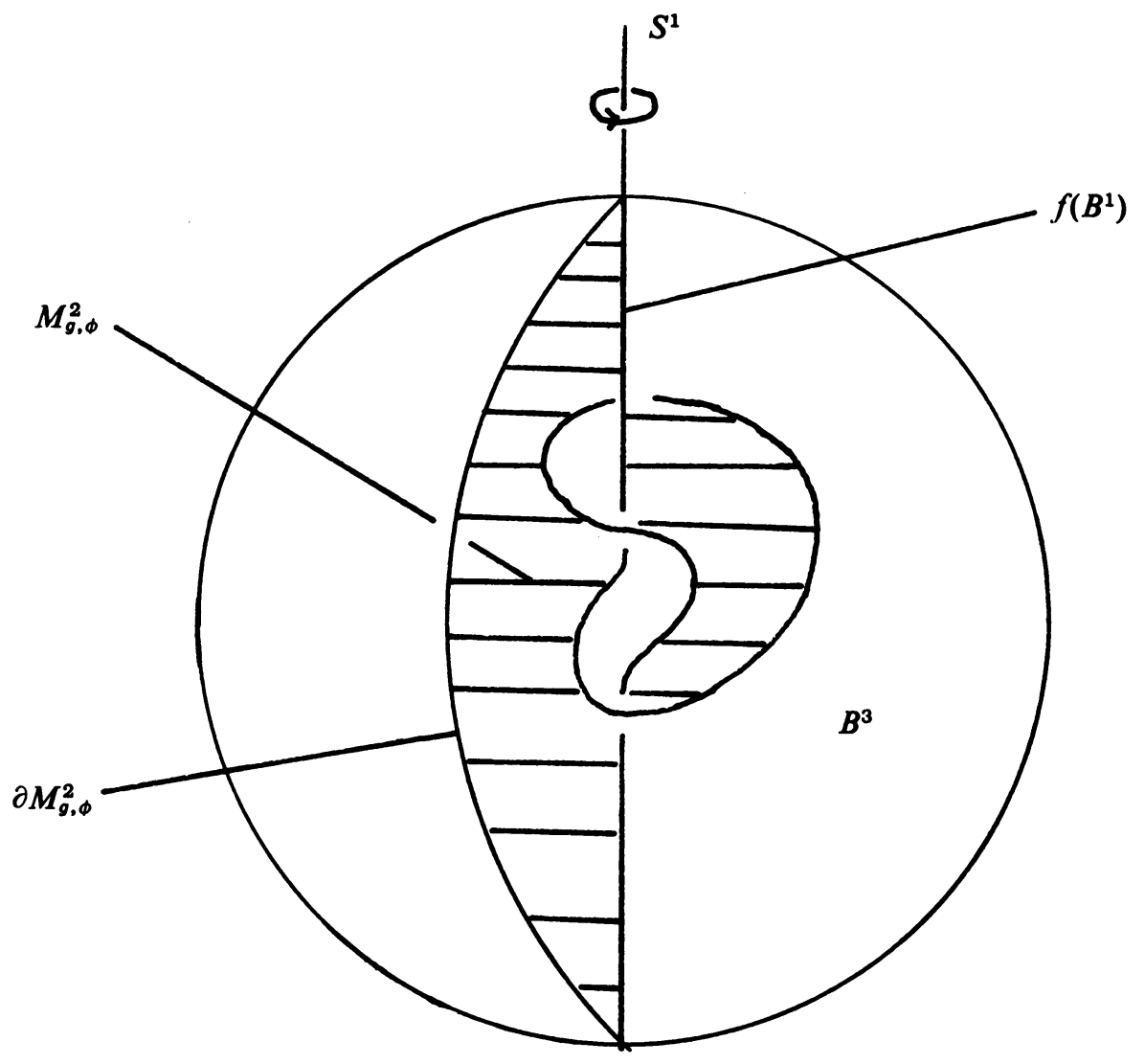

FigURE 2. $g=1$.

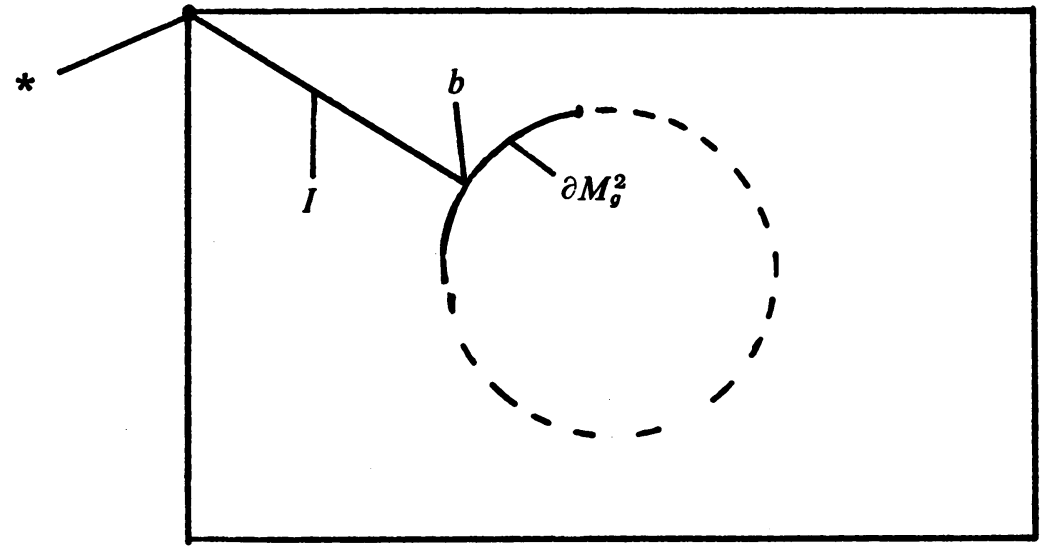

FIGURE 3. $g=1$. 
The spun fiber $F_{g, \phi}$ of $S^{k+3}-K^{k+1}$ at time $\phi$ is the following:

$$
F_{g, \phi}=\left(S^{k} \times M_{g, \phi}^{2}\right) \underset{S^{k} \times \partial M_{g, \phi}^{2}}{\left.\bigcup^{k+1} \times \partial M_{g, \phi}^{2}\right) .}
$$

Let $L_{2 g}^{p}=S^{p} \vee \cdots \vee S^{p}$ the $2 g$-fold wedge product of $S^{p}$ with itself. Now $M_{g}^{2}$ deformation retracts to $\partial M_{g}^{2} \cup I \cup L_{2 g}^{1}$ where $I$ (Figure 2) is an arc in $M_{g}^{2}$ running from a point $b \in \partial M_{g}^{2}$ to $*$ the wedge point of $L_{2 g}^{1}$ [Figure 3].

$\partial M_{g}^{2}$ deformation retracts to $b$. These deformations produce a deformation retraction from $F_{g}$ to $F_{g}^{\prime}=\left(S^{k} \times L_{2 g}^{1}\right) \cup_{S^{k} \times *}\left(B^{k+1} \times *\right)$ [Figure 4].

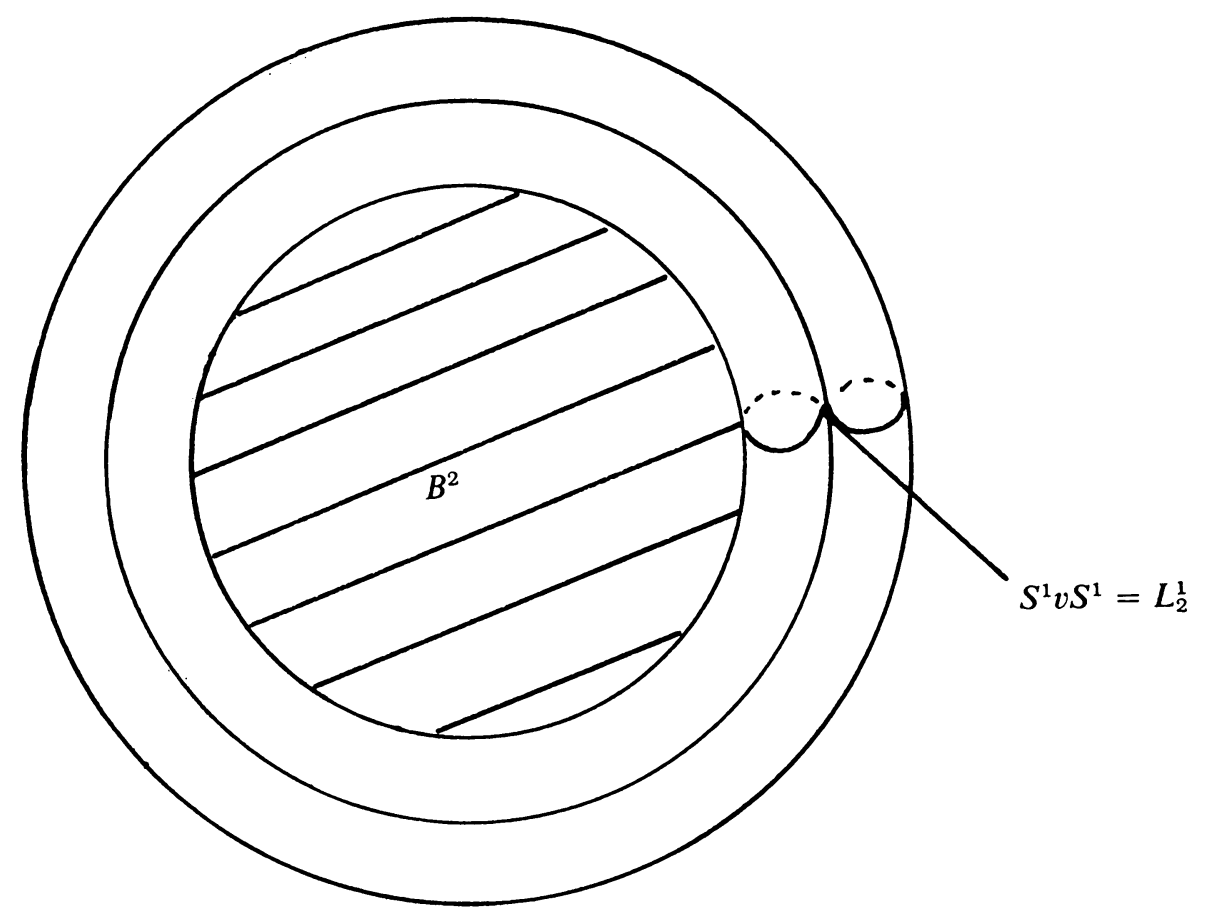

FIGURE 4. $k=1, g=1$.

Consider now $P=\left(S^{k} \times S^{1}\right) \cup S^{k} \times *\left(B^{k+1} \times *\right)$. Thinking of $S^{1}$ as the union of two arcs $I_{1}$ and $I_{2}$, we have by shrinking $I_{1}$ to a point that $P \simeq P^{\prime}$, where $P^{\prime}=\left(S^{k} \times S^{1}\right)$ $\cup_{S^{k} \times I_{1}}\left(B^{k+1} \times I_{1}\right)$ [Figure 5].

Now $P^{\prime} \simeq S^{1} \vee S^{k+1}$ by collapsing $B^{k+1} \times I_{1}$ to $I_{1}$, and then shrinking $I_{2}$ to a point. Hence $F_{g}^{\prime} \simeq L_{2 g}^{1} \vee L_{2 g}^{k+1}$. Since a homotopy equivalence of CW complexes induces a homotopy equivalence of their universal covers, we have that $\tilde{F}_{g} \simeq\left(L_{2 g}^{1} \vee L_{2 g}^{k+1}\right)^{\sim}$, the latter obtained (up to homotopy type) by taking the universal cover of $L_{2 g}^{1}$ (a snowflake with four $g$ arcs emanating from each vertex) and attaching a copy of $L_{2 g}^{k+1}$ to each vertex. Now $\left[\Pi_{1}(S), \Pi_{1}(S)\right] \cong \Pi_{1}\left(M_{g}^{2}\right) \cong \Pi_{1}\left(L_{2 g}^{1}\right)$ 
$=$ free group on $2 g$ generators. By squeezing the 1-skeleton to a point, we have $\tilde{S} \simeq V^{+} \vee \cdots \vee V^{+}$the $2 g$-fold wedge product of $V^{+}$with itself. This completes the proof of Theorem 6 .

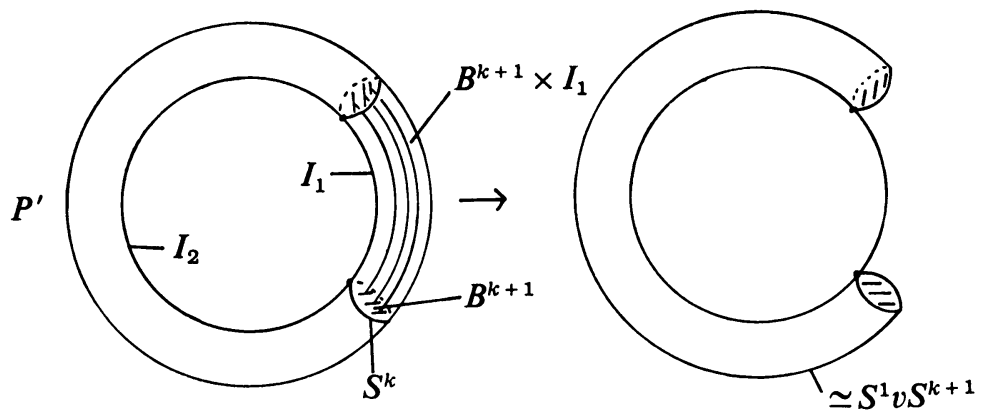

Figure 5

In [1], Andrews and Curtis ask the following question: If $\left(S^{4}, K^{2}\right)$ is the 1-spun trefoil, and $S=S^{4}-K^{2}$, is $\Pi_{3}(S)=0$ ? Theorem 5 answers this question in the negative.

CoROllary 6. Let $\left(S^{k+3}, K^{k+1}\right)$ be a $k$-spun Neuwirth knot of genus $g \geqq 1$. Then $\tilde{S} \simeq \bigvee_{i=1}^{\infty} S_{i}^{k+1}$ the infinite wedge of $k+1$-spheres.

Proof. $V^{+} \vee \cdots \vee V^{+}(2 g$ times $)=\bigvee_{i=1}^{\infty} S_{i}^{k+1}$ for all $g \geqq 1$.

This means that if we $k$-spin any two nontrivial Neuwirth knots, the universal covers of the resulting knot complements are homotopy equivalent. In fact, this also is true for iterated $k$-spinning ( $k$ allowed to vary) of Neuwirth knots, and will be dealt with in a future paper.

Corollary. Let $\left(S^{k+3}, K^{k+1}\right)$ be a k-spun Neuwirth knot of genus $g$. Then $\Pi_{k+1}(S)$ is a free $Z G$-module on $2 g$ generators.

Proof. Select a lift of $L_{2 g}^{k+1}$ to $\widetilde{F}_{g}$. Each of the spheres in the lift is a free generator of

$$
H_{k+1}\left(\tilde{F}_{g}\right) \cong Z_{G} \Pi_{k+1}\left(F_{g}\right) \cong \Pi_{k+1}(S) .
$$

The above shows that if $\left(S^{k+3}, K^{k+1}\right)$ is a $k$-spun Neuwirth knot of genus $g$, then $\Pi_{k+1}(S)$ is the free abelian group on $2 g$ copies of the symbols in $\left\{\left[\Pi_{1}(S)\right.\right.$, $\left.\left.\Pi_{1}(S)\right]\right\}$. Compare the result of Epstein [4], which says that in this case $\Pi_{k+1}(S)$ is the free abelian group on the symbols $\left\{\left[\Pi_{1}(S), \Pi_{1}(S)\right]-\mathrm{id}\right\}$. (There is in this case a 1-1 correspondence between $\left\{\left[\Pi_{1}(S), \Pi_{1}(S)\right]-\mathrm{id}\right\}$ and $2 g$ copies of $\left[\Pi_{1}(S), \Pi_{1}(S)\right]$.)

IV. Change of rings. Let

$$
1 \longrightarrow H \stackrel{\alpha}{\longrightarrow} G \underset{\gamma}{\stackrel{\beta}{\rightleftarrows}} T \longrightarrow 1
$$


be an exact sequence of groups such that $\beta \gamma=1_{T}$. Then $G$ is the semidirect product of $H$ and $T$ so that each element of $G$ can be written uniquely as $h t$ where $h \in \alpha(H)$ $=H$ and $t \in \gamma(T)=T$. Now given a $Z H$-module $A$ the group $Z G \otimes z_{H} A$ can be considered as a $Z G$-module under the action $g \cdot\left(g^{\prime} \otimes a\right)=g g^{\prime} \otimes a$ where $g, g^{\prime} \in Z G$ and $a \in A$. Also if we assume $T$ acts on $A$ as a group of automorphisms then $A$ can also be considered as a $Z G$-module under the action $g a=(h t) a=h(t a)$ where $t \in T$, $h \in H, a \in A$ and $g=h t \in G$. If $T$ is finitely generated by, say, $t_{1}, \ldots, t_{k}$ and $A$ is finitely generated as a $Z H$-module by $\bar{X}_{1}, \bar{X}_{2}, \ldots, \bar{X}_{n}$ then the action of $T$ on $A$ may be given by a matrix $\tau$ coming from $t_{p}\left(\bar{X}_{i}\right)=\sum \lambda_{i}^{p} \bar{X}_{j}$. Let $\tau$ be the $(n k+n)$ matrix

$$
\begin{gathered}
t_{1} I-\left[\lambda_{i j}^{1}\right] \\
\vdots \\
t_{k} I-\left[\lambda_{i j}^{k}\right]
\end{gathered}
$$

where $I$ is the $(n \times n)$ identity matrix, and $\left[\lambda_{i j}^{p}\right]$ is the $(n \times n)$ matrix of the coefficients.

We shall in this section use the same symbols for a map and the associated matrix given by some selection of generators. Content will make the meaning clear.

Lemma 8. Suppose $K \stackrel{\imath}{\rightarrow} M \stackrel{\phi}{\rightarrow} A \rightarrow 0$ is an exact sequence of $R$-modules with $M$ finitely presented and $K$ finitely generated. Let $\psi$ be a presentation matrix for $M$. Then a presentation matrix for $A$ is $\left(\begin{array}{l}\underset{\tau}{*} \\ )\end{array}\right)$.

Proof. Let $F_{2} \stackrel{\psi}{\rightarrow} F_{1} \stackrel{\sigma^{\prime}}{\rightarrow} A \rightarrow 0$ be a presentation of $M$ by free finitely generated $R$-modules $F_{1}, F_{2}$ and let $F \stackrel{\sigma}{\rightarrow} K \rightarrow 0$ be a map of a free finitely generated $R$ module $F$ onto $K$. We have the following diagram

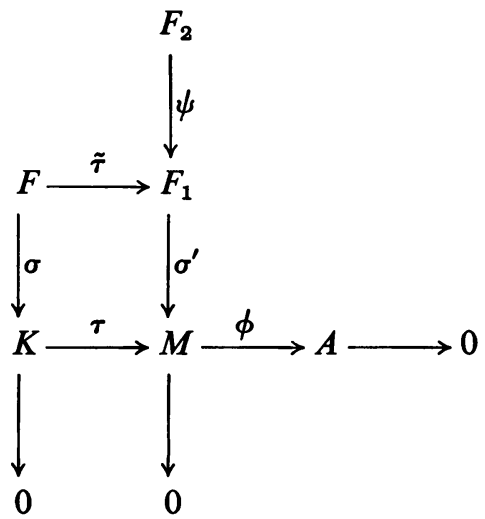

Since $F$ is projective there is a map $\tilde{\tau}: F \rightarrow F_{1}$ such that $\sigma^{\prime} \tilde{\tau}=\tau \sigma$. It follows that $\operatorname{Ker} \phi \sigma^{\prime}=\operatorname{Im} \tilde{\tau}+\operatorname{Im} \psi$ so that matrix associated with this presentation of $A$ is $\left(\begin{array}{l}\boldsymbol{\nu} \\ \tilde{\tau}\end{array}\right)$. But the matrix $\tilde{\tau}=\tau$.

THEOREM 9. Given

$$
0 \longrightarrow H \stackrel{\alpha}{\longrightarrow} G \underset{\gamma}{\stackrel{\beta}{\rightleftarrows}} T \longrightarrow 0
$$


an exact sequence of groups with $T$ finitely generated and $\beta \gamma=1_{T}$. Let $\psi$ be a presentation matrix for a finitely presented $Z$ H-module $A$ on which $T$ acts as a group of automorphism. Then $A$ is a ZG-module with presentation matrix $\left(\begin{array}{l}\psi \\ \tau\end{array}\right)$ where $\tau$ is as in (1).

Proof. Let

$$
F_{2} \stackrel{\psi}{\longrightarrow} F_{1} \stackrel{\phi}{\longrightarrow} A \longrightarrow 0
$$

be an appropriate presentation of $A$. We have the exact sequence of $Z G$-modules

$$
Z G \otimes_{Z_{H}} F_{2} \stackrel{1 \otimes \psi}{\longrightarrow} Z G \otimes_{Z_{H}} F_{1} \stackrel{1 \otimes \phi}{\longrightarrow} Z G \otimes_{Z_{H}} A \longrightarrow 0 .
$$

Let $A^{\prime}$ be the set $A$ with the $Z G$-structure obtained from the action of $Z H$ and $T$ on $A$. Then there is a natural map $\alpha: Z G \otimes z_{H} A \rightarrow A^{\prime}$ given by $\alpha(\lambda \otimes X)=\lambda X$. Clearly the kernel $K$ of $\alpha$ is generated by all elements of the form $\{\lambda \otimes X-1 \otimes(\lambda X)\}$ where $(\lambda X)$ is the element of $A$ corresponding to $\lambda X$ in $A^{\prime}$. $\left(A^{\prime}=A\right.$, setwise.) Now $K$ is generated by $\left\{t_{p} \otimes \phi\left(X_{i}\right)-1 \otimes \sum_{j} \phi_{i j}^{p} X_{j}\right\}$ for all $p, i$. That is, $K$ is generated by the images of $\left\{t_{p} \otimes X_{i}-1 \otimes \sum \lambda_{i j} X_{j}\right\}$ under $1 \otimes \phi$. The desired presentation now follows from Lemma 8.

V. Applications. Let $Q^{n+2}$ be an $(n+2)$-manifold which fibers over $S^{1}$ with fiber an $(n+1)$-manifold $F^{n+1}$. Let $p: Q \rightarrow S^{1}$ be the fiber map. We think of $Q$ as the product $F \times I$ with $F \times 0$ and $F \times 1$ identified by a homeomorphism $\hat{h}: F \times 1$ $\rightarrow F \times 0$ given by $\hat{h}(X, 1)=(h(X), 0)$. We can without loss of generality assume $h(q)=q$ for some $q \in F$. Let $t^{\prime}: I \rightarrow q \times I \subset F \times I$ be the product path from $q \times 1$ to $q \times 0$ in $F \times I$. Then (under $h) t^{\prime}$ corresponds to an element $t \in \Pi_{1}(Q, q \times 1)$ which maps onto a generator of $\Pi_{1}\left(S^{1}\right)$ under the fiber map $p_{*}$. From the homotopy exact sequence of the fibration, this gives us a semidirect splitting $\Pi_{1}(S)$ $\cong \Pi_{1}(F) \times Z$. If $i$ denotes the inclusion $i: F \rightarrow Q$ by $i(x)=(x, 1)$ and $\beta \in \Pi_{k}(F, q)$ we then have $t \cdot i_{*}(\beta)=i_{*}\left(h_{*} \beta\right)$.

We have the following corollary to Theorem 9:

CoROllaRY 10. Suppose $\Pi_{k}(F)$ is finitely presented as a $Z \Pi_{1}(F)$-module by a matrix $M$. Then $\Pi_{k}(Q) \cong \Pi_{k}(F) k \geqq 2$ is presented as a $Z \Pi_{1}(Q)$-module by $\left({ }_{t-h_{*}}\right)$, where $t-h_{*}$ denotes the appropriate matrix.

Consider the infinite cyclic cover $Q^{*}$ of $Q$. We think of $Q^{*}$ in two different ways. First as the product $Q^{*} \approx F \times R$, and second as the union of $X_{i}=(F \times I)_{i}(i \in Z)$ with $(F \times 1)_{i}$ identified with $(F \times 0)_{i+1}$ by the homeomorphism $h$. Now $t$ acts on $H_{k}\left(Q^{*}\right) \cong H_{k}(F)$ by $t(u)=h_{*}(u)$ where $u \in H_{k}(F)$ and $h_{*}: H_{k}(F) \rightarrow H_{k}(F)$ is the automorphism induced by $h$. Let $\Lambda=$ the group ring of the infinite cyclic group generated by $t$.

COROllary 11. Suppose $H_{k}(F)$ is finitely presented as an abelian group by a matrix $M$. Then $H_{k}\left(Q^{*}\right) \cong H_{k}(F)$ is presented as a $\Lambda$-module by $\left({ }_{t}-_{*}\right)$. 
Now assume $S$ and $F$ are as in $\S$ I. That is, $S$ is the complement of $K^{n}$ in $S^{n+2}$ which fibers over $S^{1}$ with fiber $F^{n+1}$.

THEOREM 12. Suppose that $G=\Pi_{1}(F)$ is finite, and that $S$ is a simple fibered knot. Then $\Pi_{n}(F)$ is presented as a ZG-module by the $(1 \times 1)$ matrix $\left(\sum_{g \in G} g\right)$.

Proof. Take $F$ to be compact and bounded with $\partial F \approx K$. Then the universal cover $\tilde{F}$ is a compact contractible manifold with $\partial \tilde{F}=\bigcup_{g \in G} K_{g}$, as in the proof of Corollary 3. By Lefschetz Duality, $H_{n}(\widetilde{F}, \partial \widetilde{F}) \cong H^{1}(\widetilde{F})=0$, and the homology exact sequence of the pair $(\tilde{F}, \partial \widetilde{F})$ yields

$$
H_{n+1}(\tilde{F}, \partial \widetilde{F}) \stackrel{\partial}{\longrightarrow} H_{n}(\partial \widetilde{F}) \longrightarrow H_{n}(\tilde{F}) \longrightarrow 0 .
$$

$H_{n+1}(\tilde{F}, \partial \widetilde{F})$ is free abelian on 1 generator $\xi$, and $H_{n}(\partial \widetilde{F})$ is free $Z G$-module on 1 generator $\alpha$, and $\partial \xi=\sum_{g \in G} g \alpha$. By Lemma 8, the result follows.

THEOREM 13. Suppose $\left(S^{n+2}, K^{n}\right)$ is a simple fibered knot, and that $G=\Pi_{1}(F)$ $=\left[\Pi_{1}(S), \Pi_{1}(S)\right]$ is finite, then $\Pi_{n}(S)$ is presented as a $Z \Pi_{1}(S)$-module by the $(2 \times 1)$-matrix

$$
\left(\begin{array}{l}
\sum_{g \in G} g \\
t-1
\end{array}\right) .
$$

Proof. We can without loss of generality replace the complement $S^{n+2}-K$ by the closed complement $S$, a manifold-with-boundary in which the entire manifold $S$ fibers over $S^{1}$ with fiber $F$, and the fibration induced on $\partial S$ has fiber $\partial F \approx K$. Moreover, up to homotopy, the fibering on the boundary is the product bundle, because any orientation preserving homeomorphism $h: K \rightarrow K$ is homotopic to the identity. We can take the homotopy class of the embedded sphere $K$ as the generator for $\Pi_{n}(F)$ as a $Z G$-module. Clearly then $h_{*}$ is the identity automorphism on $\Pi_{n}(F)$.

We are left with the case of simple fibered knots whose commutator subgroups are not finite. The general case, unfortunately, seems not so clear as the special case of $k$-spun Neuwirth knots treated earlier in §III. We do, however, have the following corollary to Theorem 1 :

CoROllary 14. $\left(S^{n+2}, K^{n}\right)$ a simple fibered knot, and $G=\Pi_{1}(S)$ is infinite. Then $\Pi_{n}(S)$ is free abelian, and $\Pi_{n}(S) \cong Z G \oplus H_{n}(\widetilde{F}, \partial \widetilde{F})$.

Proof. We have

$$
\Pi_{n}(\widetilde{S}) \cong \Pi_{n}(F) \cong Z_{G} H_{n}(\widetilde{F}) \cong Z_{G} H_{n}(\partial \widetilde{F}) \oplus H_{n}(\widetilde{F}, \partial \widetilde{F}) \cong Z_{G} Z G \oplus H_{n}(\widetilde{F}, \partial \widetilde{F}) .
$$

$H_{n}(\tilde{F})$ is free abelian, because $F^{n+1}$ is a manifold-with-boundary, hence $F \simeq X$ an $n$-dimensional finite simplicial complex. Now $H_{n}(\tilde{X}) \cong Z_{n}(\tilde{X}) \subset C_{n}(\tilde{X})$, so $H_{n}(\tilde{X})$ is free abelian. Note that $H_{n}(\tilde{F}, \partial \tilde{F}) \cong H_{n}(F, \partial F ;\{Z G\}) \cong H^{1}(F ;\{Z G\}) \cong H^{1}(G ; Z G)$ where $\{Z G\}$ denotes local coefficients and the last isomorphism is due to the fact that $\tilde{F}$ is simply connected. 
VI. Examples. Many examples of simple knots may be generated by Zeeman's twist-spinning methods [7]. For example, one can obtain fibered knots of $S^{2}$ in $S^{4}$ by twist-spinning the bridge knots. The fiber obtained is the punctured lens space $L(p, g)$ ( $p$ odd) [7, Corollary 5].

EXAMPLE 1. 5-twist-spun trefoil.

By 5-twist-spinning the trefoil, we obtain the knot $\left(S^{4}, K^{2}\right)$ and the fibration $F^{3} \rightarrow S=S^{4}-K^{2} \rightarrow S^{1}, F^{3}=$ punctured dodecahedral space. From Zeeman [7], we have that

$$
\begin{aligned}
\Pi_{1}(S) & =\left(x, y, t \mid x^{5}=(x y)^{3}=(x y x)^{2}, t^{-1} x t=y, t^{-1} y t=y x^{-1}\right), \\
G & =\Pi_{1}(F)=\left(x, y \mid x^{5}=(x y)^{3}=(x y x)^{2}\right) .
\end{aligned}
$$

In the presentation for $\Pi_{1}(S)$, the generator $t$ corresponds to the generator of $\Pi_{1}\left(S^{1}\right)$. Now $G$ is the binary dodecahedral group of order 120 . By Corollary 4 , the universal cover $\tilde{F}$ is homotopy equivalent to the wedge product of 1192 -spheres. ( $\tilde{F}$ is a 3-sphere punctured 120 times.) By Theorem $14, \Pi_{2}(S)$ is presented as a $Z \Pi_{1}(S)$-module by the $(2 \times 1)$-matrix

$$
\left(\begin{array}{l}
\sum_{g \in G} g \\
t-1
\end{array}\right) .
$$

Since $F$ is a homology 3-ball, all the Alexander invariants of the infinite cyclic cover $S^{*}$ are trivial by Corollary 11 .

EXAMPLE 2. 2-twist-spun trefoil.

This knot fibers with fiber punctured $L(3,1)$. In this case $\Pi_{1}(S)=(a, b \mid a b a$ $\left.=b a b b=a^{2} b a^{-2}\right) \cong\left(u, t \mid u^{3}=1 t u t^{-1}=u^{2}\right) . \quad G=\Pi_{1}(F)=\left(u \mid u^{3}=1\right)$. The isomorphism can be realized by setting $a b^{-1}=u, a=t$. In the second presentation for $\Pi_{1}(S), t$ represents the generator of $\Pi_{1}\left(S^{1}\right)$. Exactly as before, $\Pi_{2}(S)$ is presented as a $Z \Pi_{1}(S)$-module by the $(2 \times 1)$-matrix $\left(\begin{array}{c}1+u+u^{2} \\ t-1\end{array}\right)$. The universal cover $F$ is homotopy equivalent to $S^{2} \vee S^{2} . H_{1}\left(S^{*}\right)$ is presented as a $\Lambda$-module by the $(2 \times 1)$-matrix $\left(t_{-2}^{3}\right)$.

EXAMPLE 3. $k$-spun trefoil.

See [2], [4]. The fundamental group of the trefoil is $\left(t, u, v: t u t^{-1}=v u, t v t^{-1}\right.$ $=v u^{-1} v^{-1}$ ) where $t$ goes onto the homology generator, and $u, v$ generate the homology of the Seifert surface.

Now if $Z$ is the Seifert surface and $p$ the base point we choose $\tilde{p}$ in the universal cover $\tilde{Z}$ of $Z$ so that $\tilde{p}$ projects onto $p$. Any other point over $p$ say $\tilde{p}_{g}$ is a translation of $\tilde{p}$ by an element $g \in \pi_{1}(Z)$. If $u, v \in \pi_{1}(Z)$ are represented by maps $u, v:(I, I)$ $\rightarrow(Z, p)$ we obtain paths $\tilde{u}, \tilde{v}: I \rightarrow \tilde{Z}$ covering $u, v$ and such that $\tilde{u}(0)=\tilde{v}(0)=\tilde{p}$. Under the action of $\pi_{1}(Z)$ we obtain paths $\tilde{u}_{g}$, $\tilde{v}_{g}$ for each $g \in \pi_{1}(Z)$. When $Z$ is $k$-spun in order to obtain $F$ we may also spin $\tilde{Z}$ and obtain $\tilde{F}$. Now $\tilde{u}_{g}$ and $\tilde{v}_{g}$ give rise to $k+1$-spheres $\tilde{U}_{g}^{*}$ and $\tilde{V}_{g}^{*}$ which map to $g U^{*}$ and $g V^{*}$ in $\pi_{k}(F)$. 
In order to discover the action of $t$ on these $(k+1)$-spheres we need only note that $t$ gives rise to a homeomorphism $h: Z \rightarrow Z$ which induces $\tilde{h}: \tilde{Z} \rightarrow \tilde{Z}$ so that $\tilde{u} \rightarrow \tilde{u} \tilde{v}_{u}$ and $\tilde{v} \rightarrow \tilde{v} \tilde{u}_{u}-1 \tilde{v}_{u}{ }^{-1} v^{-1}$. These spin and project to $U^{*}+u V^{*}$ and $\left(1-u^{-1} v\right) V^{*}$ $-u^{-1} U^{*}$ in $\pi_{k+1}(F)$. So that $t\left(U^{*}\right)=U^{*}+u V^{*}$ and $t\left(V^{*}\right)=\left(1-u^{-1} v\right) V^{*}-u^{-1} U^{*}$. Hence we have a presentation matrix

$$
\left(\begin{array}{cc}
t-1 & -u \\
u^{-1} & t+v u v^{-1}-1
\end{array}\right)
$$

for $\pi_{k+1}(S)$. This is the same as that obtained by Andrews-Lomonaco [2].

\section{REFERENCES}

1. J. J. Andrews and M. L. Curtis, Knotted 2-spheres in the 4-sphere, Ann. of Math. (2) 70 (1959), 565-571. MR 21 \#5964.

2. J. J. Andrews and S. J. Lomonaco, The second homotopy group of spun 2-spheres in 4-space, Bull. Amer. Math. Soc. 75 (1969), 169-171. MR 38 \#3863.

3. W. Browder and J. Levine, Fibering manifolds over a circle, Comment. Math. Helv. 40 (1966), 153-160. MR 33 \#3309.

4. D. B. A. Epstein, Linking spheres, Proc. Cambridge Philos. Soc. 56 (1960), 215-219. MR 22 \#8514.

5. F. T. Farrell, The obsiruction to fibering a manifold over a circle, Ph.D. Thesis, Yale University, New Haven, Conn., 1968.

6. E. Spanier, Algebraic topology, McGraw-Hill, New York, 1966. MR 35 \#1007.

7. E. C. Zeeman, Twisting spun knots, Trans. Amer. Math. Soc. 115 (1965), 471-495. MR 33 \#3290.

FLorida STATE UNIVERSITY,

TALlahassee, Florida 32306 